\title{
Families of Distributions Arising from Distributions of Ordered Data
}

\author{
Mosayeb Ahmadi $^{a}$, M. Razmkhah ${ }^{b}$, G. R. Mohtashami Borzadaran ${ }^{1, b}$ \\ ${ }^{a}$ Department of Mathematics and Computer Sciences, Damghan University, Iran \\ ${ }^{b}$ Department of Statistics, Ferdowsi University of Mashhad, Iran
}

\begin{abstract}
A large family of distributions arising from distributions of ordered data is proposed which contains other models studied in the literature. This extension subsume many cases of weighted random variables such as order statistics, records, $k$-records and many others in variety. Such a distribution can be used for modeling data which are not identical in distribution. Some properties of the theoretical model such as moment, mean deviation, entropy criteria, symmetry and unimodality are derived. The proposed model also studies the problem of parameter estimation and derives maximum likelihood estimators in a weighted gamma distribution. Finally, it will be shown that the proposed model is the best among the previously introduced distributions for modeling a real data set.
\end{abstract}

Keywords: Beta distribution, location-scale family, weighted distribution, order statistics, $k$-record statistics.

\section{Introduction}

There are various types of ordered data such as usual order statistics, records and $k$-records, which come from a certain stochastic model. Moreover, there are situations in which the recoded observations do not have original distribution, while the generalized version of a certain stochastic model may be adequate for fitting the data. For example, Jones (2004) proposed a collection of generalized distributions with the probability density function (pdf)

$$
g(x) \propto F^{a}(x) \bar{F}^{b}(x) f(x)
$$

where $f(\cdot), F(\cdot)$ and $\bar{F}(\cdot)$ denote the pdf, cumulative distribution function (cdf) and survival function of the original distribution, respectively. It is clear that for the special case of $a=i-1$ and $b=n-i$, the model (1.1) simplifies to the pdf of usual order statistics. This generalization is useful in distribution theory and its empirically studying related to exploring properties of new families. The main point of Jones (2004) was to provide further families of distribution to use in modeling skewness or heavy tailed data by having $a, b$ and $f(\cdot)$. Recently, this model has also been considered by some authors. For example, Pescim et al. (2010), Silva et al. (2010), Moraisa et al. (2013) and Parnaiba et al. (2011) have discussed the advantage and disadvantage of the "beta generalized half normal", "modified Weibull", "generalized logistic" and "Burr XII" distributions, respectively. Gusmao et al. (2011)

\footnotetext{
${ }^{1}$ Corresponding author: Department of Statistics, Ferdowsi University of Mashhad, P. O. Box 1159, Mashhad 91775, Iran.

E-mail: grmohtashami@um.ac.ir,gmb1334@yahoo.com
}

Published 31 March 2015 / journal homepage: http://csam.or.kr

(c) 2015 The Korean Statistical Society, and Korean International Statistical Society. All rights reserved. 
concentrated their attention on the generalized inverse inverse Weibull distribution. Lemonte (2014) derived a new continuous distribution as a weighted version of log-logistic called the beta log-logistic with some special cases. The "beta Gumbel", "beta Fréchet" and "beta generalized exponential" are also some other famous distributions which have been investigated by Nadarajah and Kotz (2004), Nadarajah and Gupta (2004) and Barreto-Souza et al. (2010), respectively.

It is important to note that the above mentioned models are weighted versions of an original pdf. The concept of weighted distributions started by Fisher (1934). The model has been formulated in a general form by Rao (1965) for modeling the observed data which is useful in practical works. Suppose that the pdf of random variable $X$ is $f(x, \theta)$ and $w(x)$ is a non-negative function, then, the observed random variable $X_{w}$ has weighted distribution with density $g(x, \theta)=(w(x) / E[w(X)]) f(x, \theta)$. Notice that the weight function may depend on some parameters including $\theta$. The weighted distributions can be considered as a tool to select a suitable model for observed data. The pdf of order statistics, $k$-record values and also the models like truncated distribution, proportional (reversed) hazard, probability weighted models and skewed distributions are famous examples of weighted distributions. For more details see papers due to weighted models from Rao (1965) to Bartoszewicz (2009) and the books by Reiss (1989), Arnold et al. (1998), David and Nagaraja (2003), Arnold et al. (2008) and references. Recently, Ye et al. (2012) studied the weighted generalized beta distribution of the second type and related distributions such as weighted version of generalized gamma, beta of second kind, Singh-Maddala, Dagum, gamma, Weibull and exponential distributions with their applications. Furthermore, Cordeiro et al. (2012) and Ramadan (2013) investigated the class of beta extended Weibull and weighted Weibull distributions with discussions on revealing their importance in distribution theory, respectively.

Another family of weighted distributions as different version of Jones model with the pdf

$$
g(x) \propto F^{a-1}(x)\left(1-F^{a}(x)\right)^{b} f(x)
$$

is well-known as Kumaraswamy (KW) model. For various choices of $F(x)$ such as Pareto, gamma, Weibull, Gamble, inverse Gaussian, half normal, beta and generalized gamma, the Kumaraswamy model can be used to fit skewed observed data sets.

Recently, a family of distributions generated by the distribution of record statistics studied by Amini et al. (2014). This paper introduces a large family of distributions arising from distributions of ordered data which contains other models studied in the literature. The goal of this generalization is to provide more flexible families of distributions for modeling observed data. Some properties of the theoretical model are derived and it will be shown that the model is useful for modeling the data sets which are not identical in distribution.

The rest of the paper is organized as follows. In Section 2, the proposed model is described. Some properties of the model are presented in Section 3. In this section, the location-scale family of distributions are considered. The moments, two deviation criteria, entropy, symmetry and unimodality of the model are also investigated. The parameter estimates of the model is studied in Section 4 and a real data set is used to illustrate the results of the paper in Section 5.

\section{Model Description}

Numerous classical distributions have been extensively used in view of extensions of distributions of the order statistics such as Jones (2004) model with lots of motivations and distributions that are introduced. Extended class of distributions are also provided by Amini et al. (2014) including the distributions of record values and $k$-records. Here, we generalize these two ideas to obtain a family 
of distributions that subsume both of the above mechanism. Let us denote the cdf and pdf of a continuous random variable $X$ by $F(\cdot)$ and $f(\cdot)$, respectively, then a generalized class of distributions can be defined with the following pdf

$$
g_{F}(x ; a, b, c, d)=\frac{1}{M(a, b, c, d)} F^{a-1}(x) \bar{F}^{b-1}(x)(-\log F(x))^{c-1}(-\log \bar{F}(x))^{d-1} f(x),
$$

where $a, b, c$ and $d$ are positive real constant and

$$
M(a, b, c, d)=\int_{0}^{1} u^{a-1}(1-u)^{b-1}(-\log u)^{c-1}(-\log (1-u))^{d-1} d u
$$

is normalizing constant. It is clear that $(2.1)$ is a weighted distribution with weight function

$$
w(x)=F^{a-1}(x) \bar{F}^{b-1}(x)(-\log F(x))^{c-1}(-\log \bar{F}(x))^{d-1}
$$

and $E[w(X)]=M(a, b, c, d)$. Notice that the model (2.1) can be considered as a weighted version of Jones model (WJM) with weight function $w(x)=(-\log F(x))^{c-1}(-\log \bar{F}(x))^{d-1}$.

Some special cases of the WJM can be considered as:

1. Taking $c=d=1$, the proposed model leads to the Jones (2004) model, in this case for $a=i$ and $b=n-i+1$, the pdf of the $i^{\text {th }}$ order statistic in a random sample of size $n$ is derived.

2. For the special case of $b=d=1$ or $a=c=1$, the WJM leads to the Amini et al. (2014) model.

3. Taking $a=b=c=1$ and $d=m, g_{F}(x ; a, b, c, d)$ is the same as pdf of the $m^{\text {th }}$ upper record. Similarly, for $a=b=d=1$ and $c=n$, the pdf of the $n^{\text {th }}$ lower record is obtained.

4. For the case of $a=c=1, b=k$ and $d=m, g_{F}(x ; a, b, c, d)$ converts to the pdf of the $m^{t h}$ upper $k$-record. Similarly for $b=d=1, a=k$ and $c=n$, the pdf of the $n^{\text {th }}$ lower $k$-record is obtained.

5. When $b=c=d=1$ or $a=c=d=1$, the pdf of reversed proportional hazard rate model or proportional hazard rate model are derived, respectively.

In what follows, some properties of $M(a, b, c, d)$ are presented:

- (Existing) By twice use of Cauchy-Schwartz inequality we have

$$
\begin{aligned}
M(a, b, c, d) & =\int_{0}^{1} u^{a-1}(1-u)^{b-1}(-\log u)^{c-1}(-\log 1-u)^{d-1} d u \\
& \leq\left[\int_{0}^{1} u^{2(a-1)}(1-u)^{2(b-1)} d u \int_{0}^{1}(-\log u)^{2(c-1)}(-\log 1-u)^{2(d-1)} d u\right]^{\frac{1}{2}} \\
& =[\beta(2 a-1,2 b-1)]^{\frac{1}{2}}\left[\int_{0}^{\infty}\left[-\log \left(1-e^{-y}\right)\right]^{2 c-2} y^{2 d-2} e^{-y} d y\right]^{\frac{1}{2}} \\
& \leq[\beta(2 a-1,2 b-1)]^{\frac{1}{2}}\left[\int_{0}^{\infty}\left[-\log \left(1-e^{-y}\right)\right]^{4 c-4} e^{-y} d y \int_{0}^{\infty} y^{4 d-4} e^{-y} d y\right]^{\frac{1}{4}} \\
& =\sqrt{\beta(2 b-1,2 b-1)}[\Gamma(4 c-3) \Gamma(4 d-3)]^{\frac{1}{4}},
\end{aligned}
$$


where $\beta(\cdot, \cdot)$ and $\Gamma(\cdot)$ are the complete beta and gamma functions, respectively, which both are finite. Therefore, for any positive real values of $a, b, c$ and $d$ such that $a, b>1 / 2$ and $c, d>3 / 4$, the normalizing constant in (2.2) exists.

- (Expansion) For real positive value of $b$ and $d$ and integer positive values $a$ and $c$ that $c \geq 1$, by transforming $y=-\log (1-u)$ and using the identity $\log \left(1-e^{-y}\right)=-\sum_{i=1}^{\infty}\left(e^{-i y} / i\right)$, we can write

$$
\begin{aligned}
M(a, b, c, d) & =\int_{0}^{\infty}\left(1-e^{-y}\right)^{a-1}\left(e^{-y}\right)^{b-1}\left[-\log \left(1-e^{-y}\right)\right]^{c-1} y^{d-1} e^{-y} d y \\
& =\int_{0}^{\infty} y^{d-1}\left[\sum_{i=0}^{a-1}\left(\begin{array}{c}
a-1 \\
i
\end{array}\right)(-1)^{i} e^{-i y}\right] e^{-b y}\left[\sum_{i=1}^{\infty} \frac{e^{-i y}}{i}\right]^{c-1} \\
& =\sum_{i=0}^{a-1} \sum_{j=c-1}^{\infty}\left(\begin{array}{c}
a-1 \\
i
\end{array}\right)(-1)^{i} C_{j}(c-1) \int_{0}^{\infty} y^{d-1} e^{-(i+j+b) y} d y \\
& =\Gamma(d) \sum_{i=0}^{a-1} \sum_{j=c-1}^{\infty} \frac{\left(\begin{array}{c}
a-1 \\
i
\end{array}\right)(-1)^{i} C_{j}(c-1)}{(i+j+b)^{d}},
\end{aligned}
$$

where $C_{j}(n)$ is the coefficients of $w^{j}$ in the expansion of $\left(\sum_{i=1}^{\infty}\left(w^{i} / i\right)\right)^{n}$. That is, for $j \geq 1, C_{j}(1)=$ $1 / j$ and for $j \geq n \geq 2, C_{j}(n)=\sum_{k=n-1}^{j-1}\left\{C_{k}(n-1) /(j-k)\right\}$.

- If $c$ and $d$ are integer values, we can write $M(a, b, c, d)=\left(\partial^{c+d-2} \beta(a, b)\right) /\left(\partial a^{c-1} \partial b^{d-1}\right)$.

- It can be shown that for positive real values $a, b, c$ and $d$, normalizing constant is symmetric, i.e., $M(a, b, c, d)=M(b, a, d, c)$.

- In Table 1, we have presented some distributions as special cases of (2.1), which have been recently investigated by many authors.

Some special cases of $M(a, b, c, d)$ would be considered as:

1. $M(a, b, 1,1)=\beta(a, b)$,

2. $M(1,1, c, 1)=\Gamma(c)$,

3. $M(1,1,1, d)=\Gamma(d)$,

4. $M(a, 1, c, 1)=\frac{\Gamma(d)}{a^{c}}$.

Example 1. Suppose that the underlying population has exponential distribution with the $\operatorname{cdf} F(x)=$ $1-e^{-\lambda x}$. Then, for $a=1$ and $c=2$, the model (2.1) reduces to the following pdf

$$
g_{F}^{*}(x ; b, d, \lambda)=\frac{\lambda^{d}}{M(1, b, 2, d)} x^{d-1} e^{-\lambda b x}\left[-\log \left(1-e^{-\lambda x}\right)\right], \quad x>0,
$$

where

$$
M(1, b, 2, d)=\Gamma(d) \sum_{i=1}^{\infty} \frac{1}{i(b+i)^{d}} .
$$

Notice that a distribution with the pdf presented in (2.4) can be considered as a weighted gamma (WG) distribution. Figure 1 shows the pdf and cdf plots of WG model for some choices of $b, d$ and $\lambda$. 
Table 1: Some special cases of $g_{F}(x)$.

\begin{tabular}{|c|c|c|c|c|c|c|}
\hline$a$ & $b$ & $c$ & $d$ & baseline cdf & Name of Model & Reference \\
\hline$a$ & $b$ & 1 & 1 & $F(x)$ & Jones model & Jones (2004) \\
\hline$a$ & $b$ & 1 & 1 & $1-e^{-\lambda x}, \quad x>0$ & Beta exponential & Nadarajah and Kotz (2004) \\
\hline$a$ & $b$ & 1 & 1 & $\Phi(x), \quad x \varepsilon R$ & Beta Gumbel & Nadarajah and Gupta (2004) \\
\hline$a$ & $b$ & 1 & 1 & $e^{-\left(\frac{\sigma}{x}\right)^{\lambda}}, \quad x>0$ & Beta Fréchet & Barreto-Souza et al. (2010) \\
\hline$a$ & $b$ & 1 & 1 & $\exp \left\{e^{-\frac{x-\mu}{\sigma}}\right\}, \quad x, \mu \epsilon R, \sigma>0$ & Beta Gumbel & Nadarajah and Kotz (2004) \\
\hline$a$ & $b$ & 1 & 1 & $\frac{2}{\sqrt{\pi}} \Gamma\left(\frac{3}{2}, \frac{x^{2}}{2 a^{2}}\right), \quad x>0$ & Beta Maxwell & Amusan (2010) \\
\hline$a$ & $b$ & 1 & 1 & $1-\left(\frac{x}{\theta}\right)^{k}, \quad x \geq \theta, \theta, k>0$ & Beta Pareto & Akinsete et al. (2008) \\
\hline$a$ & $b$ & 1 & 1 & $1-e^{-\frac{x}{2}}, \quad x \geq 0$ & Beta Laplace & Cordeiro and Lemonte (2011) \\
\hline$a$ & $b$ & 1 & 1 & $1-\left[1+\left(\frac{x}{s}\right)^{C}\right]^{-k}$ & Beta Burr XII & Paranaiba et al. (2011) \\
\hline$a$ & $b$ & 1 & 1 & $2 \Phi\left[\left(\frac{x}{\theta}\right)^{\alpha}\right]-1$ & Beta generalized half-normal & Pescim et al. (2010) \\
\hline$a$ & $b$ & 1 & 1 & $\Phi\left(\frac{\log x-\mu}{\sigma}\right)$ & Beta log-normal & Castellares et al. (2013) \\
\hline$a$ & $b$ & 1 & 1 & $1-\exp \left\{-(\lambda x)^{c}\right\}$ & Beta extended Weibull & Cordeiro et al. (2012) \\
\hline$a$ & $b$ & 1 & 1 & $\frac{x^{\beta-1}}{\alpha^{\beta}+x^{\beta}}, \quad \alpha, \beta, x>0$ & Beta log-logistic & Lemonte (2014) \\
\hline$a$ & $b$ & 1 & 1 & $\frac{2}{\pi} \arctan (\exp (x))$ & Beta-Hyperbolic secant & Fischer and Vaughan (2010) \\
\hline$a$ & 1 & $c$ & 1 & $F(x)$ & Log-Gamma generated family & Amini et al. (2014) \\
\hline 1 & $b$ & 1 & $d$ & $F(x)$ & Log-Gamma generated family & Amini et al. (2014) \\
\hline
\end{tabular}
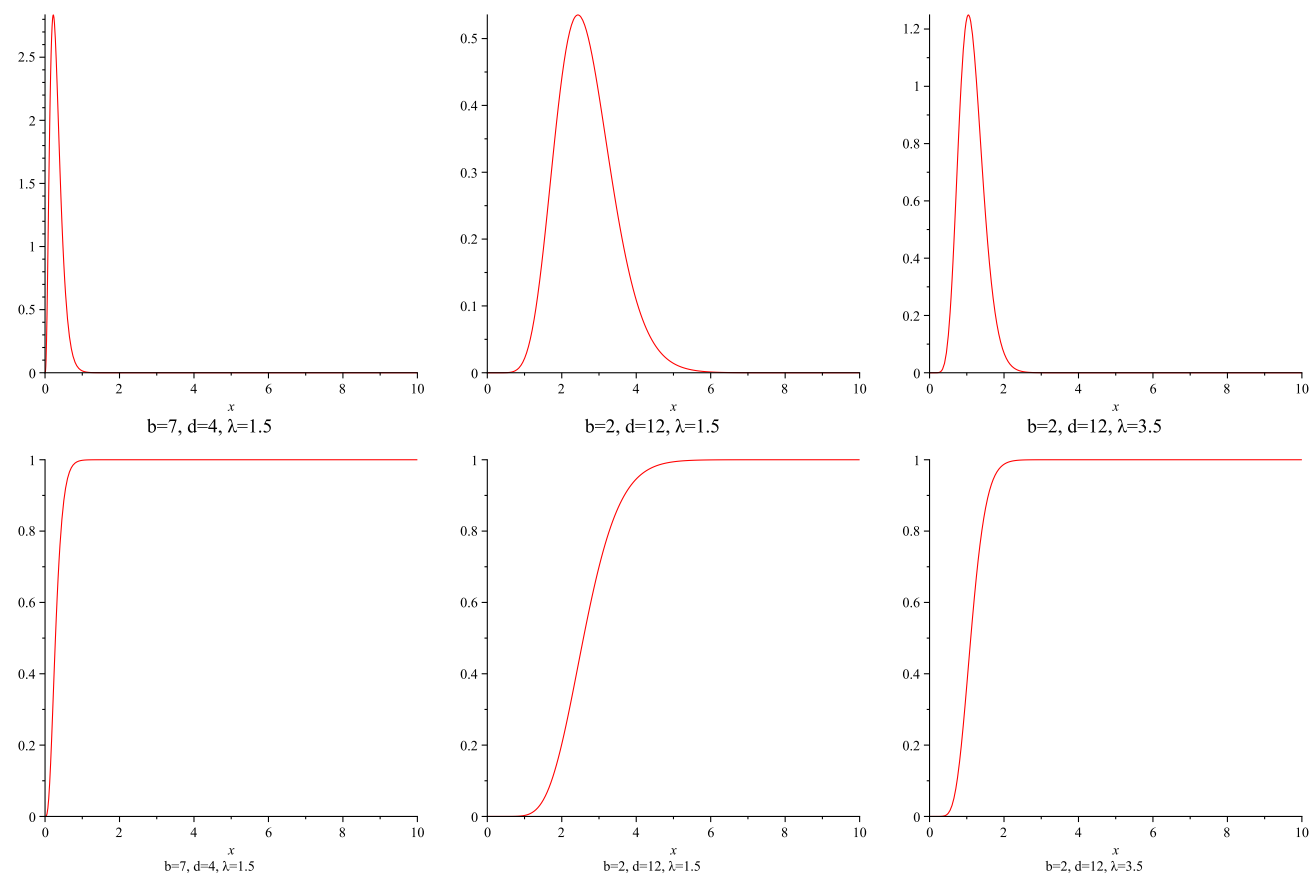

Figure 1: The pdfs (top line plots) and cdfs (low line plots) of WG model for some choices of $b, d$ and $\lambda$. 


\section{Some Properties of the Weighted Jones Model (WJM)}

In this section, some properties of the WJM are studied. First the functional form of the corresponding cdf is derived. Then, we show that this model preserves the location and scale parameter. Investigation about moments, mean deviation, symmetry, modality and Entropy criterion is also performed.

\subsection{Distribution function}

From (2.1) by change variable $u=F(x)$, the corresponding cdf of the WJM can be obtained as

$$
G_{F}(x ; a, b, c, d)=\frac{M(a, b, c, d,-\log \bar{F}(x))}{M(a, b, c, d)},
$$

where

$$
M(a, b, c, d, t)=\int_{0}^{t} u^{a-1}(1-u)^{b-1}(-\log u)^{c-1}(-\log (1-u))^{d-1} d u .
$$

For real positive values $b$ and $d$ and integer positive values $a$ and $c$, for which $c>1$, by transforming $y=-\log \bar{F}(x)$, we can write

$$
\begin{aligned}
G_{F}(x ; a, b, c, d) & =\frac{1}{M(a, b, c, d)} \int_{0}^{-\log \bar{F}(x)}\left(1-e^{-y}\right)^{a-1}\left(e^{-y}\right)^{b-1}\left[-\log \left(1-e^{-y}\right)\right]^{c-1} y^{d-1} e^{-y} d y \\
& =\frac{\sum_{i=0}^{a-1} \sum_{j=c-1}^{\infty} \frac{\left(\begin{array}{c}
a-1 \\
i
\end{array}\right)(-1)^{i} C_{j}(c-1)[\Gamma(d)-\Gamma(d,-(i+j+b) \log \bar{F}(x))]}{(i+j+b)^{d}}}{\Gamma(d) \sum_{i=0}^{a-1} \sum_{j=c-1}^{\infty} \frac{\left(\begin{array}{c}
a-1 \\
i
\end{array}\right)(-1)^{i} C_{j}(c-1)}{(i+j+b)^{d}}} \\
& =1-\frac{\sum_{i=0}^{a-1} \sum_{j=c-1}^{\infty} \frac{\left(\begin{array}{c}
a-1 \\
i
\end{array}\right)(-1)^{i} C_{j}(c-1) \Gamma[d,-(i+j+b) \log \bar{F}(x)]}{(i+j+b)^{d}}}{\Gamma(d) \sum_{i=0}^{a-1} \sum_{j=c-1}^{\infty} \frac{\left(\begin{array}{c}
a-1 \\
i
\end{array}\right)(-1)^{i} C_{j}(c-1)}{(i+j+b)^{d}}}
\end{aligned}
$$

where $\Gamma(d, t)=\int_{t}^{\infty} y^{d-1} e^{-y} d y$ is the incomplete gamma function. Note that known $F(\cdot)$ and $(a, b, c, d)$ implies via (3.3) random numbers with distribution $g_{F}(\cdot)$.

Furthermore, using (3.3), it is deduced that the corresponding cdf of (2.4) is given by

$$
G_{F}(x ; b, d, \lambda)=1-\frac{\sum_{j=1}^{\infty} \frac{\Gamma[d,(\lambda b+\lambda j) x]}{j(b+j)^{d}}}{\Gamma(d) \sum_{j=1}^{\infty} \frac{1}{j(j+b)^{d}}} .
$$

\subsection{Location-scale family}

Let the underlying cdf belong to the location-scale family of distribution, i.e., $F(x ; \mu, \sigma)=F_{0}((x-\mu) /$ $\sigma)$. In this case, the parameters $\mu$ and $\sigma$ are also of great interest and the pdf in (2.1) can be extended to the following parameterized pdf

$$
\begin{aligned}
& g_{F}(x ; a, b, c, d, \mu, \sigma) \\
& =\frac{1}{M(a, b, c, d)} F_{0}^{a-1}\left(\frac{x-\mu}{\sigma}\right) \bar{F}_{0}^{b-1}\left(\frac{x-\mu}{\sigma}\right)\left[-\log F_{0}\left(\frac{x-\mu}{\sigma}\right)\right]^{c-1} \times\left[-\log \bar{F}_{0}\left(\frac{x-\mu}{\sigma}\right)\right]^{d-1} \frac{1}{\sigma} f\left(\frac{x-\mu}{\sigma}\right) \\
& =\frac{1}{\sigma} g_{F_{0}}\left(\frac{x-\mu}{\sigma} ; a, b, c, d, 0,1\right) \\
& =\frac{1}{\sigma} g_{F_{0}}\left(\frac{x-\mu}{\sigma} ; a, b, c, d\right) .
\end{aligned}
$$


It is observed that if the underlying distribution belong to location-scale family, then WJD, also is, i.e., this model preserves the location and scale parameter.

\subsection{Moments}

Here, we first study the necessary condition for the existence of the moments of the WJM, then present some exact expressions for the situations in which the parent distribution is exponential.

Lemma 1. Let $X$ be a random variable with $c d f F(x)$ and $p d f f(x)$ and $X_{*}$ be a random variable with $p d f(2.1)$. If $E\left(|X|^{k+\delta}\right)<\infty$ for any non-negative integer $k$ and $\delta>0$, then for $a, b>1 / 2$ and $c, d>3 / 4, E\left(\left|X_{*}\right|^{k}\right)<\infty$.

Proof: By the use of Holder and Cauchy-Schwartz inequalities, we get

$$
\begin{aligned}
E\left(\left|X_{*}\right|^{k}\right) & =\frac{1}{M(a, b, c, d)} \int_{-\infty}^{\infty}\left|x_{*}\right|^{k}\left[F\left(x_{*}\right)\right]^{a-1}\left[\bar{F}\left(x_{*}\right)\right]^{b-1}\left[-\log F\left(x_{*}\right)\right]^{c-1}\left[-\log \bar{F}\left(x_{*}\right)\right]^{d-1} f\left(x_{*}\right) d x_{*} \\
& =\frac{1}{M(a, b, c, d)} \int_{0}^{1}\left|F^{-1}(u)\right|^{k} u^{a-1}(1-u)^{b-1}(-\log u)^{c-1}(-\log (1-u))^{d-1} d u \\
& \leq \frac{\left[\int_{0}^{1}(-\log u)^{q(c-1)}(-\log (1-u))^{q(d-1)} d u\right]^{\frac{1}{q}}\left[\int_{0}^{1}\left|F^{-1}(u)\right|^{p k} u^{p(a-1)}(1-u)^{p(b-1)} d u\right]^{\frac{1}{p}}}{M(a, b, c, d)}\left[\int_{0}^{1}\left|F^{-1}(u)\right|^{p k} u^{p(a-1)}(1-u)^{p(b-1)} d u\right]^{\frac{1}{p}} \\
& =\frac{[M(1,1, c q-q+1, d q-q+1)]^{\frac{1}{q}}}{M(a, b, c, d)}\left[\int_{0}^{1}\left|F^{-1}(u)\right|^{2 p k} d u \int_{0}^{1} u^{2 p(a-1)}(1-u)^{2 p(b-1)} d u\right]^{\frac{1}{2 p}} \\
& \leq \frac{[M(1,1, c q-q+1, d q-q+1)]^{\frac{1}{q}}}{M(a, b, c, d)} \\
& =\frac{[M(1,1, c q-q+1, d q-q+1)]^{\frac{1}{q}}[\beta(2 a p-2 p+1,2 p b-2 p+1)]^{\frac{1}{2 p}}}{M\left(E|X|^{k+\delta}\right]^{\frac{1}{2 p}}, \quad(3.5)}
\end{aligned}
$$

where $2 k p=k+\delta$. Hence, the proof is complete.

Now, consider the situation in which $c$ and $d$ are integers and the parent distribution is standard exponential. Then, the $k^{\text {th }}$ moment of $X_{*}$ is given by

$$
\begin{aligned}
E\left(X_{*}^{k}\right) & =\int_{0}^{1} u^{a-1}(1-u)^{b-1}(-\log (u))^{c-1}(-\log 1-u)^{k+d-1} d u \\
& =\frac{\partial^{c+d+k-2} \beta(a, b)}{\partial a^{c-1} \partial b^{k+d-1}}\left(\frac{\partial^{c+d-2} \beta(a, b)}{\partial a^{c-1} \partial b^{d-1}}\right)^{-1} .
\end{aligned}
$$

Based on (3.6), the measures of skewness (SK) and kurtosis (KU) of WJM can be obtained as

$$
\mathrm{SK}=\frac{\frac{\Delta(c-1, d+2)}{\Delta(c-1, d-1)}-3 \frac{\Delta(c-1, d)}{\Delta(c-1, d-1)} \frac{\Delta(c-1, d+1)}{\Delta(c-1, d-1)}+2\left[\frac{\Delta(c-1, d)}{\Delta(c-1, d-1)}\right]^{3}}{\left[\frac{\Delta(c-1, d+1)}{\Delta(c-1, d-1)}-\left(\frac{\Delta(c-1, d)}{\Delta(c-1, d-1)}\right)^{2}\right]^{\frac{3}{2}}}
$$



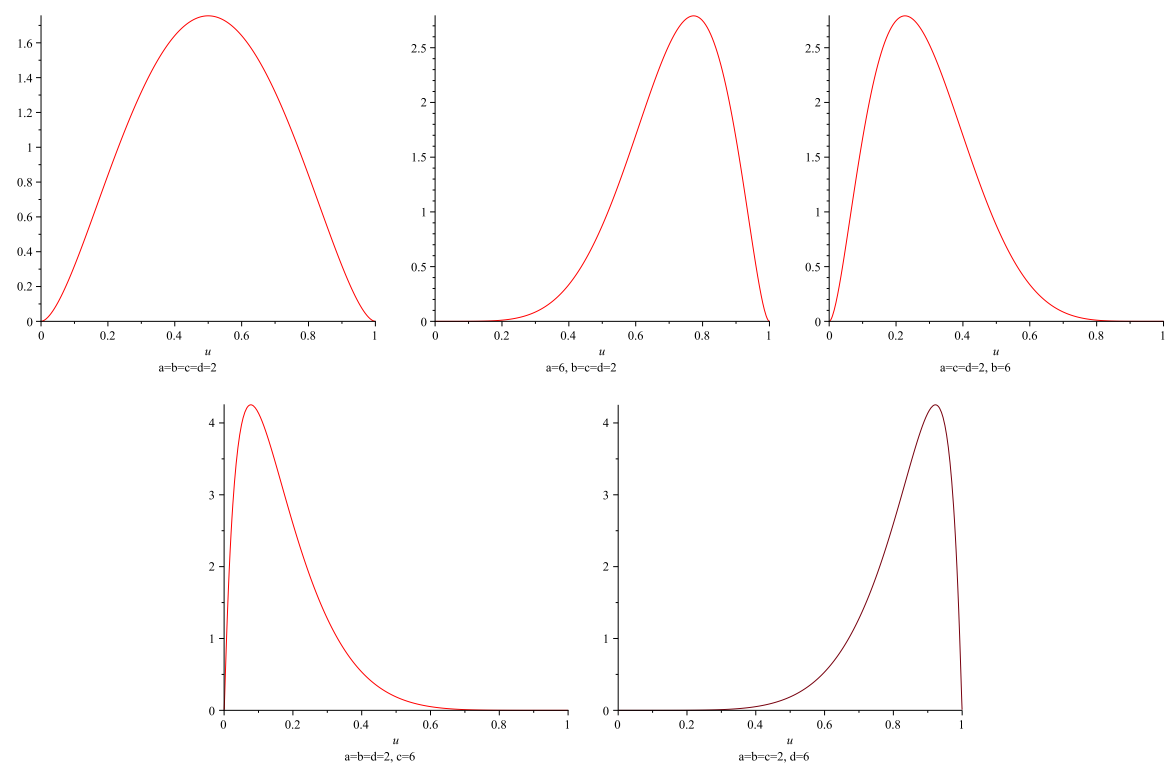

Figure 2: The plots of $g_{F}(\cdot)$, when the baseline distribution is standard uniform with various values of $a, b, c$ and $d$

and

$$
\mathrm{KU}=\frac{\frac{\Delta(c-1, d+3)}{\Delta(c-1, d-1)}-4 \frac{\Delta(c-1, d)}{\Delta(c-1, d-1)} \frac{\Delta(c-1, d+2)}{\Delta(c-1, d-1)}+6\left(\frac{\Delta(c-1, d)}{\Delta(c-1, d-1)}\right)^{2} \frac{\Delta(c-1, d+1)}{\Delta(c-1, d-1)}-3\left(\frac{\Delta(c-1, d)}{\Delta(c-1, d-1)}\right)^{4}}{\left[\frac{\Delta(c-1, d+1)}{\Delta(c-1, d-1)}-\left(\frac{\Delta(c-1, d)}{\Delta(c-1, d-1)}\right)^{2}\right]^{2}}-3,
$$

respectively, where $\Delta(i, j)=\partial^{i+j} \beta(a, b) /\left(\partial a^{i} \partial b^{j}\right)$.

For the special case of WG distribution with pdf in (2.4), the moment generating function is given by

$$
\begin{aligned}
M(t) & =\frac{\lambda^{d}}{M(1, b, 2, d)} \int_{0}^{\infty} x^{d-1} e^{-(\lambda b-t) x}\left(-\log \left(1-e^{-\lambda x}\right)\right) d x \\
& =\frac{\lambda^{d}}{M(1, b, 2, d)} \sum_{i=1}^{\infty} \frac{\Gamma(d)}{i(\lambda b+\lambda i-t)^{d}},
\end{aligned}
$$

moreover, it can be shown that the $r^{\text {th }}$ moment is

$$
\mu_{r}=\frac{\lambda^{d}}{M} \int_{0}^{\infty} x^{d+r-1} e^{-\lambda b x} \sum_{i=1}^{\infty} \frac{e^{-i \lambda x}}{i} d x=\frac{\Gamma(d+r)}{\lambda^{r} M(1, b, 2, d)} \sum_{i=1}^{\infty} \frac{1}{i(i+b)^{r+d}},
$$

where $M(1, b, 2, d)$ is defined by (2.5).

Remark 1. If $a \neq b$ or $c \neq d$ and the baseline distribution be symmetric, then $g_{F}$ will be skew. Note that the value of skewness depends on the difference $b-a$ and $c-d$. Moreover, the sign of skewness depends on sign of $(b-a)+(c-d)$. Figure 2 shows the plots of $g_{F}(\cdot)$, when the baseline distribution is standard uniform with various values of $a, b, c$ and $d$. 


\subsection{Mean deviations}

As we know in symmetric distributions, deviation from the mean, and in skewed distributions, deviation from the median can be used as a measure of spread in a population. Let $X$ be random variable with pdf $f(x)$ and cdf $F(x)$, then, the mean deviation from the mean and the mean deviation from the median are defined by

$$
\delta_{1}(X)=\int_{-\infty}^{\infty}|x-\mu| f(x) d x
$$

and

$$
\delta_{2}(X)=\int_{-\infty}^{\infty}|x-m| f(x) d x,
$$

respectively, where $\mu=E(X)$ and $m$ is the median of $X$. As mentioned in Nadarajah et al. (2011), we have

$$
\delta_{1}(x)=2 \mu F(\mu)-2 \mu+2 \int_{\mu}^{\infty} x f(x) d x
$$

and

$$
\delta_{2}(x)=2 \int_{m}^{\infty} x f(x) d x-\mu .
$$

To calculate the above quantities for random variable $X_{*}$ with pdf (2.1), we use the following identity

$$
\begin{aligned}
\int_{l_{1}}^{l_{2}} x g_{F}(x) d x & =\frac{1}{M(a, b, c, d)} \int_{F\left(l_{1}\right)}^{F\left(l_{2}\right)} F^{-1}(u) u^{a-1}(1-u)^{b-1}(-\log u)^{c-1}(-\log (1-u))^{d-1} d u \\
& =K\left[F\left(l_{1}\right), F\left(l_{2}\right), a, b, c, d\right], \quad \text { say. }
\end{aligned}
$$

Hence

$$
\begin{aligned}
\int_{\mu_{*}=E_{g}\left(X_{*}\right)}^{\infty} x g_{F}(x) d x & =\frac{1}{M(a, b, c, d)} \int_{F\left(\mu_{*}\right)}^{1} F^{-1}(u) u^{a-1}(1-u)^{b-1}(-\log u)^{c-1}(-\log (1-u))^{d-1} d u \\
& =K\left[F\left(\mu_{*}\right), 1, a, b, c, d\right] \\
& =K[F(K(0,1, a, b, c, d)), 1, a, b, c, d] .
\end{aligned}
$$

Therefore,

$$
\begin{aligned}
\delta_{1}\left(X_{*}\right)= & 2 \mu_{*} G\left(\mu_{*}\right)-2 \mu_{*}+2 \int_{\mu_{*}}^{\infty} y g_{F}(x) d x \\
= & 2 \mu_{*} K\left[0, F\left(\mu_{*}\right), a, b, c, d\right]-2 \mu_{*}+2 K\left[F\left(\mu_{*}\right), 1, a, b, c, d\right] \\
= & 2 K[0,1, a, b, c, d] K[0, F(K(0,1, a, b, c, d)), a, b, c, d]-2 K[0,1, a, b, c, d] \\
& +2 K[F(K(0,1, a, b, c, d)), 1, a, b, c, d]
\end{aligned}
$$

and

$$
\delta_{2}\left(X_{*}\right)=2 K\left[G\left(m_{*}\right), 1, a, b, c, d\right]-K[0,1, a, b, c, d],
$$


where $m_{*}$ is the median of $X_{*}$ and derived from $(1 / M(a, b, c, d)) \int_{0}^{F\left(m_{*}\right)} u^{a-1}(1-u)^{b-1}(-\log u)^{c-1}(-\log (1$ $-u))^{d-1} d u=1 / 2$.

Example 2. For the pdf (2.4), we have $\delta_{1}\left(X_{*}\right)=2(A-1)(B-1)-2$ and $\delta_{2}\left(X_{*}\right)=A-\{2 M(1, b, 2, d+$ $1,1 / 2)\} /\{\lambda M(1, b, 2, d)\}$, where $A=M(1, b, 2, d+1) /\{\lambda M(1, b, 2, d)\}$ and $B=M(1, b, 2, d+1,1-$ $\left.e^{-\lambda A}\right) /\{\lambda M(1, b, 2, d)\}$.

Remark 2. Bonferroni index $(B(p))$ and Lorenz curve $(L(p))$ are important criteria in economic and income studies and other fields. For the random variable $Y$ with the pdf $g_{F}(y)$, they can be defined as follows $B(p)=\left(1 / p \mu_{*}\right) \int_{0}^{G^{-1}(p)} x g_{F}(x) d x$ and $L(p)=\left(1 / \mu_{*}\right) \int_{0}^{G^{-1}(p)} x g_{F}(x) d x$. Therefore, using (3.10), we get $B(p)=1 /\{p K(0,1, a, b, c, d)\} K\left[0, F\left(G^{-1}(p)\right), a, b, c, d\right]=L(p) / p$.

\subsection{Entropy}

The notion of entropy has an important role in statistics, probability and communication theory. Shannon (1948) defined the entropy in discrete version with sensitive application in communication theory. Let $X$ be continuous random variable with pdf $f(x)$, then entropy of $X$ is defined as

$$
H(X)=-\int_{-\infty}^{\infty} f(x) \log (f(x)) d x .
$$

So, for the weighted random variable $X_{w}$ with pdf $f_{w}(x)=w(x) f(x) / E(w(X))$, we have

$$
H\left(X_{w}\right)=-\frac{1}{E(w(X))}\left\{E_{f}[w(X) \log w(X)]+E_{f}[w(X) \log f(x)]\right\}+\log E(w(X)) .
$$

When the weight function is a function of parent cdf, i.e., $w(x)=\phi(F(x))$, the entropy is simplified to

$$
H\left(X_{w}\right)=-\frac{1}{A}\left[B+C_{f}\right]+\log A,
$$

where

$$
\begin{aligned}
& A=E_{f}[w(X)]=\int_{0}^{1} \phi(y) d y \\
& B=E_{f}[w(X) \log w(X)]=\int_{0}^{1} \phi(y) \log \phi(y) d y
\end{aligned}
$$

and

$$
C_{f}=\int_{0}^{1} \phi(y) \log f\left(F^{-1}(y)\right) d y .
$$

Remark 3. Let $X_{1}$ and $X_{2}$ be two random variables with cdfs $F_{1}$ and $F_{2}$, respectively. Also, assume that $X_{w_{i}}(i=1,2)$ be a weighted random variable of $X_{i}$ with weight function $w_{i}(x)=\phi\left(F_{i}(x)\right)$, then $H\left(X_{w_{1}}\right)=H\left(X_{w_{2}}\right)$ if $C_{f_{1}}=C_{f_{2}}$.

It is of interest to note that for model (2.1), we get

$$
\begin{aligned}
& A=M(a, b, c, d) \\
& B=(1-a) M(a, b, c+1, d)+(1-b) M(a, b, c, d+1)+(c-1) \frac{\partial M(a, b, c, d)}{\partial c}+(d-1) \frac{\partial M(a, b, c, d)}{\partial d}
\end{aligned}
$$


and

$$
C_{f}=\int_{0}^{1} y^{a-1}(1-y)^{b-1}(-\log y)^{c-1}(-\log 1-y)^{d-1} \log f\left(F^{-1}(y)\right) d y .
$$

Example 3. Let the parent distribution at the pdf (2.1) be the Weibull distribution with survival function $\bar{F}(x)=e^{-\alpha x^{\beta}}, x \geq 0$. Then, we can write

$$
\begin{aligned}
H\left(X_{*}\right)= & -\frac{1}{A}\left[B+\log \left(\beta \alpha^{\beta}\right) M(a, b, c, d)+\frac{1}{\beta} \log (\beta) M(a, b, c, d)\right. \\
& \left.-M(a, b, c, d+1)+\frac{\beta-1}{\beta} \frac{\partial M(a, b, c, d)}{\partial d}\right]+\log A .
\end{aligned}
$$

Note that $\beta=1$ and $\beta=2$ imply the exponential and Rayleigh distributions as special cases, respectively.

Lemma 2. If $F$ and $f$ are the parent $c d f$ and $p d f$ respectively and $g_{F}(\cdot)$ is the WJM, then,

(a) $E_{g_{F}}\left[(\log F(X))^{l}\right]=\frac{(-1)^{l} M(a, b, c+l, d)}{M(a, b, c, d)}$,

(b) $E_{g_{F}}\left[(\log (1-F(X)))^{\prime^{\prime}}\right]=\frac{(-1)^{l^{\prime}} M\left(a, b, c, d+l^{\prime}\right)}{M(a, b, c, d)}$,

(c) $E_{g_{F}}\left[(\log F(X))^{l}(\log (1-F(X)))^{l^{\prime}}\right]=\frac{(-1)^{l+l^{\prime}} M\left(a, b, c+l, d+l^{\prime}\right)}{M(a, b, c, d)}$.

\subsection{Symmetry and unimodality}

Let $f$ be symmetric about zero, i.e., $F(x)=\bar{F}(-x)$ and $f(x)=f(-x)$. In this case, we get

$$
\begin{aligned}
g_{F}(-x ; a, b, c, d) & =\frac{1}{M(a, b, c, d)} F(-x)^{a-1} \bar{F}(-x)^{b-1}(-\log F(-x))^{c-1}(-\log \bar{F}(-x))^{d-1} f(-x) \\
& =\frac{1}{M(a, b, c, d)} F(x)^{b-1} \bar{F}(x)^{a-1}(-\log F(x))^{d-1}(-\log \bar{F}(x))^{c-1} f(x) \\
& =g_{F}(x ; b, a, d, c) .
\end{aligned}
$$

Using (3.17), it is observed that in the special case $a=b$ and $c=d$, the pdf $g_{F}(\cdot)$ is also symmetric.

Lemma 3. Let $f(\cdot)$ be symmetric and unimodal pdf with corresponding $c d f F(\cdot)$, then $g_{F}(\cdot)$ is also unimodal with the same mode, if $a=b$ and $c=d$.

Proof: By using (2.1), the first and second derivatives of $g_{F}(\cdot)$ are

$$
g_{F}^{\prime}(x)=f(x) g_{F}(x)\left[\frac{a-1}{F(x)}-\frac{b-1}{\bar{F}(x)}+\frac{c-1}{F(x) \log F(x)}-\frac{d-1}{\bar{F}(x) \log \bar{F}(x)}\right]+\frac{f^{\prime}(x)}{f(x)} g_{F}(x),
$$

and

$$
g_{F}^{\prime \prime}(x)=g_{F}^{\prime}(x) D(x)+D^{\prime}(x) g_{F}(x)
$$


respectively, where

$$
D(x)=f(x)\left[\frac{a-1}{F(x)}-\frac{b-1}{\bar{F}(x)}+\frac{c-1}{F(x) \log F(x)}-\frac{d-1}{\bar{F}(x) \log \bar{F}(x)}\right]+\frac{f^{\prime}(x)}{f(x)}
$$

and

$$
\begin{aligned}
D^{\prime}(x)= & (a-1) \frac{f^{\prime}(x) F(x)-f^{2}(x)}{F^{2}(x)}-(b-1) \frac{f^{\prime}(x) \bar{F}(x)-f^{2}(x)}{\bar{F}^{2}(x)} \\
& +(c-1) \frac{f^{\prime}(x) F(x) \log F(x)-f^{2}(x)(1+\log F(x))}{(F(x) \log F(x))^{2}} \\
& -(d-1) \frac{f^{\prime}(x) \bar{F}(x) \log \bar{F}(x)-f^{2}(x)(1+\log \bar{F}(x))}{(\bar{F}(x) \log \bar{F}(x))^{2}}+\frac{f^{\prime \prime}(x) f(x)-\left(f^{\prime}(x)\right)^{2}}{f^{2}(x)} .
\end{aligned}
$$

Assuming $m$ is the median of the symmetric pdf $f(x)$, we get $F(m)=0.5$ and $f^{\prime}(m)=0$. Therefore, $g_{F}^{\prime}(m)=0$ and $g_{F}^{\prime \prime}(m) \leq 0$. That is, $m$ is also the mode of $g_{F}(\cdot)$.

Remark 4. In the cases that the baseline distribution is neither symmetric nor unimodal, depending on various values of $a, b, c$ and $d$, the pdf $g_{F}(\cdot)$ should be unimodal or bimodal.

\section{Estimation of Parameters}

Let the parent cdf $F$ be free of parameters and suppose $X_{1}, X_{2}, \ldots, X_{n}$ constitute a random sample of size $n$ from the pdf (2.1), then the likelihood function of the model for parameters $a, b, c$ and $d$ is given by

$$
L(a, b, c, d)=[M(a, b, c, d)]^{-n}\left(\Pi_{i=1}^{n} f\left(x_{i}\right)\right) \exp \left\{(a-1) t_{1}+(b-1) t_{2}+(c-1) t_{3}+(d-1) t_{4}\right\},
$$

where $t_{1}, t_{2}, t_{3}$ and $t_{4}$ are the observed values of $T_{1}(X)=\sum_{i=1}^{n} \log \left[F\left(X_{i}\right)\right], T_{2}(X)=\sum_{i=1}^{n} \log \left[\bar{F}\left(X_{i}\right)\right]$, $T_{3}(X)=\sum_{i=1}^{n} \log \left[-\log F\left(X_{i}\right)\right]$ and $T_{4}(X)=\sum_{i=1}^{n} \log \left[-\log \bar{F}\left(X_{i}\right)\right]$, respectively, which are complete sufficient statistics for the parameters $a, b, c$ and $d$, respectively. Using (4.1), the maximum likelihood estimators (MLE) for these parameters can be obtained by solving the following equations:

$$
\left\{\begin{array}{c}
T_{1}(X)-n \frac{\partial}{\partial a} \log M(a, b, c, d)=0 \\
T_{2}(X)-n \frac{\partial}{\partial b} \log M(a, b, c, d)=0 \\
T_{3}(X)-n \frac{\partial}{\partial c} \log M(a, b, c, d)=0 \\
T_{4}(X)-n \frac{\partial}{\partial d} \log M(a, b, c, d)=0
\end{array}\right.
$$

The above equations can be numerically solved. It is clear that if the underlying cdf belongs to the location-scale family of distribution, our model have six parameters which is complicated in general, but some special cases are noticeable. Here, we focus our attention to the WG distribution with the pdf (2.4). 
Let us now consider a random sample of size $n$ from the WG distribution with pdf (2.4). In this case, the parameter $\lambda$ may be estimated based on a random sample of size $n$ from the WG distribution. That is, $\hat{\lambda}=1 / \bar{X}$, where $\bar{X}$ is the sample mean. Hence, the log-likelihood function of the parameters $b$ and $d$ is:

$$
l(b, d, \widehat{\lambda})=-n \log M+n \log \widehat{\lambda}+(d-1) \sum_{i=1}^{n} \log X_{i}-b \widehat{\lambda} \sum_{i=1}^{n} X_{i}+\sum_{i=1}^{n} \log \left[-\log \left(1-e^{-\widehat{\lambda} X_{i}}\right)\right] .
$$

Therefore, the MLEs of $b$ and $d$ can be obtained by solving the following two equations

$$
\left\{\begin{array}{l}
-n \frac{\partial}{\partial b} \log M(1, b, 2, d)+\widehat{\lambda} \sum_{i=1}^{n} X_{i}=0 \\
-n \frac{\partial}{\partial d} \log M(1, b, 2, d)+n \log \widehat{\lambda}+\sum_{i=1}^{n} \log X_{i}=0
\end{array}\right.
$$

By doing some algebraic calculations it can be shown that

$$
\begin{aligned}
M(1, b, 2, d) & =\Gamma(d) \sum_{j=1}^{\infty} \frac{1}{j(j+b)^{d}}, \\
\frac{\partial}{\partial b} M(1, b, 2, d) & =-\Gamma(d+1) \sum_{j=1}^{\infty} \frac{1}{j(j+b)^{d+1}}, \\
\frac{\partial}{\partial d} M(1, b, 2, d) & =\Gamma(d) \sum_{j=1}^{\infty} \frac{\Psi(d)-\log (b+j)}{j(j+b)^{d}},
\end{aligned}
$$

where $\Psi(\cdot)$ is the digamma function. In the next subsection, we use the above approach to estimate the parameters of model (2.1).

\section{Application to a Real Data Set}

To illustrate the results of the paper, we use the annual flood loss data which is reported by the United States (U.S.) Army Corps of Engineers to U.S. Congress. The data, available at http://www.nws.noaa. gov/hic, were obtained by the National Weather Service (NWS) that continually gathers data from each weather forecast office, and employs a rigorous process to quality control the damage estimates. The NWS primary mission is to provide weather information for the protection of life and property. Ancillary to this mission, NWS field offices provide loss estimates for significant flood events. Annual flood loss data adjusted to 2011 inflation in \$Billion from 1950-2011 are:

$$
\begin{aligned}
& 3.13,17.18,4.05,1.85,1.54,13.68,0.85,4.51,2.61,1.61,1.02,1.65,0.78, \\
& 1.79,6.31,7.36,1.04,3.17,2.67,6.45,1.48,1.65,23.10,9.07,2.59,5.63, \\
& 11.33,4.58,2.29,10.57,4.20,2.57,5.93,8.92,8.20,1.08,12.67,2.97,0.45, \\
& 2.12,3.14,3.19,1.39,28.50,1.89,8.47,9.88,13.59,3.83,8.17,1.95,10.45, \\
& 1.68,3.36,17.81,51.17,4.38,2.97,7.63,1.06,5.19,8.41 .
\end{aligned}
$$

The Kolmogorov-Smirnov (K-S) test confirm that these data come from exponential distribution. The upper 4-records extracted from above data:

$1.85,3.13,4.05,4.51,6.31,6.45,7.36,9.07,11.33,12.67,13.68,17.18,17.81$. 
Table 2: Comparison among different models in view of both K-S test and AIC criterion.

\begin{tabular}{|c|c|c|c|c|c|}
\hline \multirow{2}{*}{ Model } & \multirow{2}{*}{ Reference } & \multirow{2}{*}{ pdf } & \multicolumn{2}{|c|}{$\mathrm{K}-\mathrm{S}$ test } & \multirow{2}{*}{ AIC } \\
\hline & & & test statistic & $p$-value & \\
\hline Weighted Gamma (WG) & Present paper & $g_{F}(\cdot ; 1, b, 2, d)$ & 0.1163 & 0.9859 & 81.0521 \\
\hline Jones model & Jones $(2004)$ & $g_{F}(\cdot ; a, b, 1,1)$ & 0.1211 & 0.9790 & 81.1974 \\
\hline Log-Gamma generated family & Amini et al. (2014) & $g_{F}(\cdot ; 1, b, 1, d)$ & 0.4352 & 0.0093 & 94.3895 \\
\hline Log-Gamma generated family & Amini et al. (2014) & $g_{F}(\cdot ; a, 1, c, 1)$ & 0.1213 & 0.9786 & 81.1959 \\
\hline
\end{tabular}

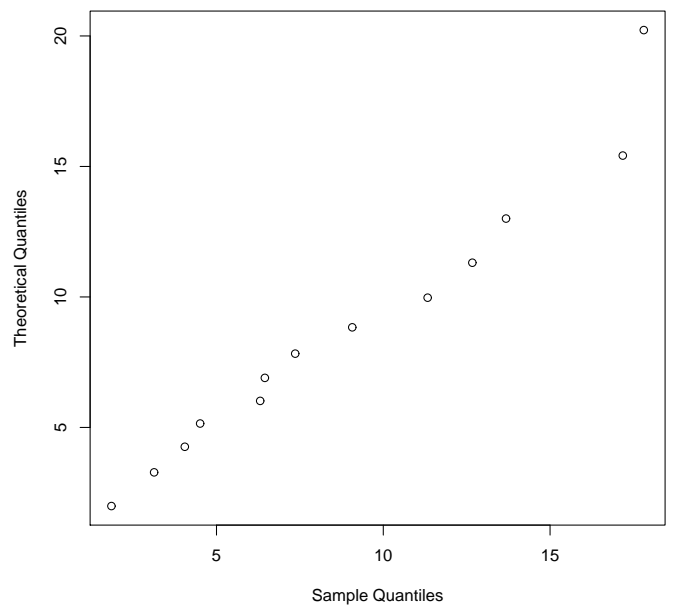

Figure 3: Q-Q plot of the observed sample against the weighted gamma distribution.

Notice that these data do not come from the same distribution, therefore, the weighted upper $k$-record model such as the weighted model (2.4) seems suitable to fit them. Using the main data, the maximum likelihood estimate of $\lambda$ is to be $\widehat{\lambda}=0.1539$. To obtained the MLEs of the parameters $b$ and $d$ based on upper 4-records, one may solve the equations (4.1), which yields $\hat{b}=1.1071$ and $\hat{d}=3.1085$. Finally, the K-S test is also used to clarify that upper 4-records come from the pdf $g_{F}^{*}(\cdot ; 1.1071,3.1085,0.1539)$. The observed value of K-S statistic is 0.1998 and the associated $p$ value is 0.6082. Moreover, the AIC for this model is equal to 81.0521. Based on these observations, one cannot reject the hypothesis that the data come from WG distribution with the pdf (2.4). For more investigations about this model and comparing with those of Jones (2004) with pdf $g_{F}(x ; a, b, 1,1)$ and Amini et al. (2014) with pdfs $g_{F}(x ; a, 1, c, 1)$ and $g_{F}(x ; 1, b, 1, d)$, a comparison has been done in view of both K-S test and AIC criterion. Table 2 presents the results and the entries of Table 2 confirm that the proposed weighted gamma distribution provides a good fit for the above data. This is also corroborated by the Q-Q plot of the sample quantiles against the quantiles of the pdf in (2.4) presented in Figure 3.

\section{Conclusions}

This paper introduced a new family of distributions containing types of ordered data called WJM. Some properties of this model were investigated; subsequently, the parameter estimation problem of was also studied briefly. As we know, besides the Jones model, upper and lower $k$-record statistics provide more information about right and left distribution tails, respectively. Nevertheless, WJM is a combination of them. Development of different fields of sciences and the need for more flexible models, led to introducing the generalized models. WJM includes many existing models on the inter- 
pretation of distribution tails.

\section{References}

Akinsete, A., Famoye, F. and Lee, C. (2008). The beta-Pareto distribution, Statistics, 42, 547-563.

Amini, M., MirMostafaee, S. M. T. K. and Ahmadi, J. (2014). Log-Gamma generated families of distributions, Statistics, 48, 913-932.

Amusan, G. E. (2010). The Beta Maxwell Distribution, Marshall University, Thesis.

Arnold, B. C., Balakrishnan, N. and Nagaraja, H. N. (2008). A First Course in Order Statistics, SIAM, Philadelphia.

Arnold, B. C., Balakrishnan, N. and Nagaraja, H. N. (1998). Records, John Wiley \& Sons, New York.

Bartoszewicz, J. (2009). On a representation of weighted distributions, Statistics and probability Letters, 79, 1690-1694.

Barreto-Souza, W., Santos, A. and Cordeiro, G. M. (2010). The beta generalized exponential distribution, Journal of Statistical Computation and Simulation, 80, 159-172.

Castellares, F., Montenegro, L. C. and Cordeiro, G. M. (2013). The beta log-normal distribution, Journal of Statistical Computation and Simulation, 83, 203-228.

Cordeiro, G. M. and Lemonte, A. J. (2011). The beta Laplace distribution, Statistics and Probability Letters, 81, 973-82.

Cordeiro, G. M., Ortega, E. M. M. and Silva, G. O. (2012). The beta extended Weibull family, Journal of Probability and Statistical Science, 10, 15-40.

David, H. A. and Nagaraja, H. N. (2003). Order Statistics, Third Edition, Wiley, New Jersey,

Fischer, M. J. and Vaughan, D. (2010). The beta-hyperbolic secant distribution, Austrian Journal of Statistics, 39, 245-258.

Fisher, R. A. (1934). The effect of methods of ascertainment upon the estimation of frequencies. Annals of Eugenics, 6, 13-25.

Gusmao, F. R. S., Ortega, E. M. M. and Cordeiro, G. M. (2011). The generalized inverse Weibull distribution, Statistical Papers, 52, 591-619.

Jones, M. C. (2004). Families of distributions arising from distributions of order statistics (with discussion), Test, 13, 1-43.

Lemonte, A. J. (2014). The beta log-logistic distribution, Brazilian Journal of Probability and Statistics, 28, 313-332.

Moraisa, A. L., Cordeiro, G. M. and Cysneiros, A. H. M. A. (2013). The Beta generalized logistic distribution, Brazilian Journal of Probability and Statistics, 27, 185-200.

Nadarajah, S., Bakouch, H. S. and Tahmasbi, R. (2011). A generalized Lindley distribution, Sankhya B, 73, 331-359.

Nadarajah, S. and Kotz, S. (2004). The beta Gumbel distribution, Mathematical Problems in Engineering, 10, 323-332.

Nadarajah, S. and Gupta, A. K. (2004). The beta Fréchet distribution, Far East Journal of Theoretical Statistics, 14, 15-24.

Parnaiba, P. F., Ortega, E. M. M., Cordeiro, G. M. and Pescim, R. R. (2011). The beta Burr XII distribution with application to lifetime data, Computational Statistics and Data Analysis, 55, 1118-1136.

Pescim, R. R., Demério, C. G. B., Cordeiro, G. M., Ortega, E. M. M. and Urbanoa, M. R. (2010). The beta generalized half-normal distribution, Computational Statistics and Data Analysis, 54, 945-957. 
Ramadan, M. M. (2013). A class of weighted Weibull distributions and its properties, Studies in Mathematical Sciences, 6, 35-45.

Reiss, R. D. (1989). Approximate Distributions of Order Statistics: With Applications to Nonparametric Statistics, Springer-Verlag, Berlin.

Rao, C. R. (1965). Weighted distributions arising out of methods of ascertainment. In Classical and Contagious Discrete Distributions, G. P. Patil (Eds). Calcutta: Pergamon Press and Statistical Publishing Society, 320-332.

Shannon, C. E. (1948). A mathematical theory of communication, Bell System Technical Journal, 27, 379-423.

Silva, G. O., Ortega, E. M. M. and Cordeiro, G. M. (2010). The beta modified Weibull, Distribution, Lifetime Data Analysis, 16, 409-430.

Ye, Y., Oluyede, B. O. and Pararai, M. (2012). Weighted generalized beta distribution of the second kind and related distributions, Journal of Statistical and Econometric Methods, 1, 13-31. 